\title{
Agravos à saúde referidos pelos trabalhadores de enfermagem em um hospital público da Bahia
}

\author{
Health problems reported by nursing workers in a public hospital of Bahia
}

Problemas de salud reportados por trabajadores de enfermería en un hospital público de Bahia

\section{Luciana Souza de Freitas Machado', Eder Pereira Rodrigues', Luciana de Matos Mota Oliveira', Rodrigo Cunha Sales Laudano', Carlito Lopes Nascimento Sobrinho'}

\author{
'Universidade Estadual de Feira de Santana, Departamento de Saúde, \\ Programa de Pós-Graduação em Saúde Coletiva. Feira de Santana-BA, Brasil.
}

Submissão: $30-01-2013$

Aprovação: 22-08-2014

\section{RESUMO}

Embora o trabalho seja essencial à vida humana, o contexto no qual é realizado pode desencadear tensão, desajuste e consequente adoecimento. Estudos apontam a Enfermagem como uma ocupação com alto risco para adoecimento, em especial no ambiente hospitalar. O objetivo deste estudo foi estimar a ocorrência de agravos à saúde referidos pelos trabalhadores de enfermagem em um hospital na Bahia. Estudo de corte transversal envolvendo 309 profissionais. As queixas de saúde mais frequentes relacionaram-se a sintomas osteomusculares como dor nas pernas 66,4\% (192) e dor nas costas 61,8\% (178); e à saúde mental com predomínio de cansaço mental 47\% (131) e nervosismo 33,7\% (93). Os resultados apontam que enfermeiros apresentaram maior frequência de agravos posturais e queixas de saúde mental enquanto as queixas de agravos respiratórios foram mais prevalentes entre técnicos e auxiliares. Estes resultados evidenciam necessidade de maior consciência do processo de trabalho e realização de atividades preventivas.

Descritores: Enfermagem; Morbidade; Saúde do Trabalhador; Epidemiologia.

\begin{abstract}
Although the work is essential to human life, the context in which it is done can induct tension, imbalance and consequent illness. Studies indicate nursing as an occupation with high risk for disease, particularly in the hospital environment. The aim of this study was to estimate the occurrence of health problems reported by nursing staff in a hospital in Bahia, Brazil. This is a cross-sectional study, involving 309 nursing professionals. The most frequent health complaints were related to musculoskeletal symptoms such as leg pain $66.4 \%$ (192) and back pain $61.8 \%$ (178); and to mental health with a predominance of mental fatigue $47 \%$ (131) 33 and nervousness $7 \%$ (93). The results show that nurses had a higher frequency of postural problems and mental health complaints while respiratory diseases complaints were more prevalent among technicians and assistants. These results highlight the need for greater awareness of the work process and implementation of preventive activities.
\end{abstract}

Key words: Nursing; Morbidity; Occupational Health; Epidemiology.

\section{RESUMEN}

Aunque el trabajo es esencial para la vida humana, el contexto en el que se realiza puede desencadenar tensión, desequilibrio y enfermedad consecuente. Los estudios muestran la enfermería como una profesión de alto riesgo para la enfermedad, sobre todo en el medio hospitalario. El objetivo de este estudio fue estimar la incidencia de problemas de salud reportados por el personal de enfermería en un hospital de Bahia, Brasil. Trata-se de un estudio transversal con 309 profesionales. Los problemas de salud más frecuentes relacionadas con los síntomas musculo esqueléticos, como el dolor de pierna 66.4\% (192) y el dolor de espalda $61,8 \%$ (178) y la salud mental, con un predominio de la fatiga mental 47\% (131) 33 y el nerviosismo $7 \%$ (93). Los resultados muestran que las enfermeras tenían una mayor frecuencia de problemas de postura y quejas de salud mental mientras que las quejas de las enfermedades respiratorias son más frecuentes entre los técnicos y auxiliares. Estos resultados ponen de relieve la necesidad de una mayor conciencia sobre el proceso de trabajo y la ejecución de las actividades preventivas.

Palabras clave: Enfermería; Morbilidad; Salud Ocupacional; Epidemiología. 


\section{INTRODUÇÃO}

O trabalho é essencial para a vida humana. A maneira como é desenvolvido associa-se às vivências objetivas e subjetivas dos trabalhadores. Nesse sentido, os agravos à saúde do trabalhador desenvolvem-se de maneira multicausal, devendo-se levar em conta a interação entre fatores patogênicos. Os estudos sobre o adoecer dos trabalhadores e as formas como ele interage com o trabalho tem ganhado destaque na comunidade científica e propiciam uma compreensão mais ampla da ocorrência e do curso dos agravos à saúde bem como das suas consequências diretas e indiretas para os indivíduos, famílias e sociedade.

Estudos apontam para o entendimento que o trabalho não é neutro em relação à saúde, uma vez que seu desenvolvimento se dá mediante múltiplas situações como forma de organização e gestão, relações e contratos de trabalho que repercutem no viver, adoecer e morrer dos trabalhadores ${ }^{(1)}$.

O trabalho de enfermagem em instituições hospitalares foi escolhido como foco deste estudo. No Brasil, os profissionais de enfermagem, em especial os que trabalham em instituições hospitalares têm reconhecidamente longas jornadas de trabalho. A escala de plantões permite que esses profissionais se dediquem a mais de uma atividade produtiva o que pode levar à exaustão e consequente adoecimento, podendo ainda afetar a qualidade da assistência aos pacientes ${ }^{(2)}$.

A assistência de enfermagem é prestada por uma equipe com formação técnica diferenciada cabendo ao enfermeiro ações gerenciais, de supervisão e prestação de cuidados de maior complexidade. Ao pessoal de nível médio cabe executar, sob supervisão, atividades menos complexas, e assistência direta ao cliente, como banho, troca de roupas, administração de medicações, como dispõe a Lei $n^{\circ} 7498$ de 25 de junho de 1986, que regulamenta o exercício da Enfermagem.

Essa divisão das atribuições torna o trabalho de enfermagem parcelado, fragmentado acrescido de elevada carga de trabalho, tensão emocional advinda do contato direto com a dor, a morte e o desenvolvimento de tarefas desagradáveis, repulsivas e aterradoras, pode gerar danos à saúde, propiciando a ocorrência de acidentes, adoecimento e morte ${ }^{(3)}$.

Com relação ao adoecimento, os trabalhadores de enfermagem apresentam perfis diferenciados da população em geral, pois se encontram mais propensos a adoecer ou morrer por causas relacionadas ao trabalho e pela exposição às cargas e riscos inerentes a sua atividade laboral.

Frente a esta situação, o objetivo deste estudo foi estimar a ocorrência de agravos à saúde, referidos pelos trabalhadores de enfermagem (auxiliares, técnicos, enfermeiros) em um hospital público de Feira de Santana, Bahia.

\section{METODOLOGIA}

Este estudo utilizou informações coletadas na pesquisa "Condições de trabalho e transtornos mentais comuns na equipe de enfermagem em um hospital geral público em Feira de Santana-BA", realizada por pesquisadores da Sala de Situação e Análise Estatística e Epidemiológica da Universidade
Estadual de Feira de Santana, no ano de 2010. Trata-se, portanto, de um recorte dos dados existentes visando abranger apenas os agravos à saúde e fatores relacionados a estes.

Trata-se de uma pesquisa exploratório-descritiva, realizada em um Hospital Geral de Feira de Santana-BA, de grande porte, centro de referência na atenção à saúde de média e alta complexidade do Sistema Único de Saúde (SUS). A população do estudo foi constituída por enfermeiros, técnicos e auxiliares de enfermagem atuantes nos diversos setores do hospital no ano de 2010. Participaram da pesquisa 309 trabalhadores: 85 enfermeiros, 233 técnicos e auxiliares de enfermagem.

Os critérios de inclusão foram: ser trabalhadores da equipe de enfermagem (enfermeiros, técnicos e auxiliares); estar desenvolvendo atividades assistenciais e aceitar participar do estudo após a leitura e assinatura do termo de consentimento livre esclarecido (TCLE). Foram excluídos do estudo aqueles que, no momento da coleta, estavam afastados do trabalho por doença, licença gestação, férias, e que não estivessem atuando em atividades assistenciais.

A coleta de dados foi realizada utilizando-se questionário padronizado respondido no próprio local de trabalho. $\mathrm{O}$ questionário levantou as características sociodemográficas; as condições de trabalho; as queixas de saúde e doença, acidentes de trabalho, problemas de saúde recentes e hábitos de vida. O questionário incluiu ainda instrumentos validados internacionalmente como o Job Content Questionnaire (JCQ). Para avaliação da saúde mental dos trabalhadores, foram utilizados os escores obtidos no Self Report Questionnaire (SRQ-20), com ponto de corte para suspeição de Sofrimento Mental (DPM) de sete ou mais respostas positivas. Como teste de triagem para detecção de abuso no consumo de bebidas alcoólicas, foi utilizado o Teste CAGE (Acrossomia referente a quatro perguntas do original em inglês: Cut down, Annoyed by cristicism, Guilty e Eye- opener), adotando-se como ponto de corte duas ou mais respostas positivas.

$\mathrm{Na}$ avaliação do padrão de sono utilizou-se o Mine - Sleep Questionnaire (MSQ), que, de uma forma abrangente, avalia aspectos relacionados ao padrão de sono, classificando-o como: sono muito bom (0-9 pontos), sono bom (10-24 pontos), sono levemente alterado (25-27 pontos), sono moderadamente alterado (28-30 pontos) e sono muito alterado (acima de 30 ).

Foi levantado inicialmente o perfil da população estudada, a partir de frequências dos dados de interesse, sendo utilizado o programa estatístico "Social Package for the Social Sciences - SPSS", versão 9.0 for Windows.

A análise descritiva buscou identificar as frequências absolutas e relativas para as variáveis categóricas. A descrição iniciou-se pela análise univariada do perfil de cada variável separadamente. A seguir, foi feita a análise da relação entre as variáveis referentes às condições do trabalho, variáveis referentes à saúde e variáveis sociodemográficas, através da análise bivariada.

Foram separadas duas categorias profissionais, a de Enfermeiros, agrupando-se a categoria de Técnicos e auxiliares de enfermagem, visto que, neste hospital, estes profissionais desenvolvem o mesmo tipo de atividade. A prevalência foi utilizada como medida de ocorrência para as variáveis de interesse. 
O estudo obteve parecer favorável do Comitê e Ética em Pesquisa da Universidade Estadual de Feira de Santana sob protocolo n. 14/2010; CAAE 09896012.6.0000.0053.

\section{RESULTADOS E DISCUSSÃO}

\section{Características sociodemográficas}

Responderam ao questionário 309 profissionais de enfermagem, correspondendo a $100 \%$ da população elegível. Dentre os entrevistados $72,4 \%$ (223) eram Técnicos e auxiliares de enfermagem e $26,6 \%$ (85) eram enfermeiros. A proporção de enfermeiros na equipe é superior a relatada no estudo realizado com profissionais de enfermagem no Mato Grosso do Sul que identificou $15,7 \%$ da equipe como enfermeiros ${ }^{(4)}$ e em estudo realizado em um hospital geral na Serra Gaúcha, onde $11,6 \%$ dos entrevistados pertenciam a esta categoria profissional $^{(5)}$.

Constatou-se que a maioria dos trabalhadores entrevistados pertence ao sexo feminino com $90,8 \%$ (278). A média de idade da população estudada foi de $36,9 \pm 9,9$, não havendo diferenças entre os grupos profissionais (Tabela 1). Este resultado reflete o fato de que, historicamente, a categoria de enfermagem tem se configurado como predominantemente feminina, razão pela qual as questões relacionadas ao gênero não podem deixar de ser consideradas na análise do processo saúde-doença dessa categoria de trabalhadores. Em especial, devem ser consideradas as interações entre o trabalho remunerado e o trabalho doméstico, geralmente atribuído às muIheres, que podem tornar-se potencializadores das cargas de trabalho, sendo este um aspecto-chave na compreensão do impacto diferenciado das condições de trabalho sobre a saúde de homens e mulheres.
Com relação à situação conjugal este estudo demonstrou que a maioria eram casados ou conviviam em união livre, condição apontada por outro estudo semelhante como mecanismo protetor ao adoecimento físico e psíquico ${ }^{(5)}$. No entanto, neste estudo essa associação não foi abordada. (Tabela 1).

No atual contexto da enfermagem os profissionais têm buscado cada vez mais qualificação. A pesquisa em questão revelou que, entre os enfermeiros, $27,1 \%$ possuem pós-graduação e, entre os técnicos e auxiliares de enfermagem, 11,5\% apresentam nível superior. Este dado é relevante, visto que a qualificação profissional pode refletir na melhora do atendimento ao paciente, na medida em que favorece o melhor desenvolvimento de suas competências (Tabela 1).

No entanto, o fato de profissionais de nível médio adquirir qualificação igual ou superior à de seus superiores imediatos, mas permanecer em cargos de menor prestígio e com salários inferiores, pode ser condição desencadeadora de conflitos nas relações de poder e de agravos de ordem psíquica entre estes profissionais, fato que requer estudos mais aprofundados.

Com relação aos hábitos de vida, 87,5\% (264) dos profissionais relataram realizar atividade de lazer, pelo menos uma vez por semana, sendo esta frequência levemente superior entre os enfermeiros $90,4 \%$ (75), quando comparado com os técnicos e auxiliares $86,3 \%$ (189). Entende-se que o lazer tem papel fundamental enquanto meio alternativo para o relaxamento e alívio dos problemas advindos do contexto e cotidiano do indivíduo (Tabela 2).

O tabagismo e etilismo foram investigados, sendo os escores mais elevados na categoria de nível médio. Entre os que informaram fazer uso de bebida alcoólica 7,5\% (04) foram positivos ao Teste CAGE e destes $100 \%$ eram técnicos e auxiliares. (Tabela 2).

Tabela 1 - Características sociodemográficas dos trabalhadores de enfermagem de um hospital público de Feira de Santana, Bahia, 2010

\begin{tabular}{|c|c|c|c|c|c|}
\hline \multirow{2}{*}{ Variável } & & \multicolumn{2}{|c|}{ Técnicos / Aux. de enfermagem } & \multicolumn{2}{|c|}{ Enfermeiros } \\
\hline & & $\mathbf{n}$ & $\%$ & $\mathbf{n}$ & $\%$ \\
\hline \multirow[t]{2}{*}{ Sexo } & Feminino & 200 & 90,9 & 77 & 90,6 \\
\hline & Masculino & 20 & 9,1 & 08 & 9,4 \\
\hline \multirow{3}{*}{ Faixa Etária } & Até 30 anos & 61 & 29,8 & 38 & 35,0 \\
\hline & 31 a 45 anos & 90 & 43,9 & 30 & 42,4 \\
\hline & Mais de 45 anos & 54 & 26,3 & 10 & 22,6 \\
\hline \multirow[t]{3}{*}{ Situação conjugal } & Casado/União livre & 104 & 49,5 & 44 & 53,0 \\
\hline & Solteiro & 80 & 38,1 & 31 & 37,3 \\
\hline & Divorciado/separado/ desquitado/Viúvo & 26 & 12,4 & 08 & 9,6 \\
\hline \multirow[t]{3}{*}{ Naturalidade } & Feira de Santana & 108 & 49,3 & 46 & 54,1 \\
\hline & Outro município & 99 & 45,2 & 32 & 37,6 \\
\hline & Outro Estado & 12 & 5,5 & 07 & 8,2 \\
\hline \multirow{2}{*}{ Ter Filhos } & Não & 81 & 37,5 & 44 & 53,0 \\
\hline & Sim & 135 & 62,5 & 39 & 47,0 \\
\hline \multirow[t]{2}{*}{ Escolaridade } & Até $2^{\circ} \mathrm{Grau}$ & 193 & 88,5 & - & - \\
\hline & Superior completo & 25 & 11,5 & 84 & 100,0 \\
\hline
\end{tabular}

* respostas válidas, excluídas as respostas ignoradas. 
Tabela 2 - Hábitos de vida dos trabalhadores de enfermagem de um hospital público em Feira de Santana, Bahia, 2010

\begin{tabular}{|c|c|c|c|c|c|}
\hline \multirow[t]{2}{*}{ Hábitos de vida } & & \multicolumn{2}{|c|}{$\begin{array}{l}\text { Técnicos / Aux. } \\
\text { de enfermagem }\end{array}$} & \multicolumn{2}{|c|}{ Enfermeiros } \\
\hline & & $\mathbf{n}$ & $\%$ & $\mathbf{n}$ & $\%$ \\
\hline Desenvolve alguma atividade de lazer & $\begin{array}{l}\text { Sim } \\
\text { Não }\end{array}$ & $\begin{array}{r}189 \\
30\end{array}$ & $\begin{array}{l}86,3 \\
13,7\end{array}$ & $\begin{array}{r}75 \\
7\end{array}$ & $\begin{array}{r}90,4 \\
9,6\end{array}$ \\
\hline Frequência com que pratica atividade de lazer & $\begin{array}{l}\text { Todos os dias } \\
3-4 \times \text { /semana } \\
1-2 \times \text { / semana }\end{array}$ & $\begin{array}{r}- \\
- \\
219\end{array}$ & $\begin{array}{r}- \\
- \\
100,0\end{array}$ & $\begin{array}{r}- \\
01 \\
82\end{array}$ & $\begin{array}{r}- \\
1,2 \\
98,8\end{array}$ \\
\hline Tipo de atividade & $\begin{array}{l}\text { Assistir TV /Ouvir música } \\
\text { Cinema / Teatro /Shows / Barzinho } \\
\text { Praia / Piscina } \\
\text { Visita/ Sair com amigos } \\
\text { Prática de esportes }\end{array}$ & $\begin{array}{r}123 \\
67 \\
10 \\
09 \\
01\end{array}$ & $\begin{array}{r}58,6 \\
31,9 \\
4,8 \\
4,3 \\
0,5\end{array}$ & $\begin{array}{l}26 \\
33 \\
12 \\
07 \\
05\end{array}$ & $\begin{array}{r}31,3 \\
39,8 \\
14,5 \\
8,4 \\
6,0\end{array}$ \\
\hline Tabagismo & $\begin{array}{l}\text { Nunca fumou } \\
\text { Ex fumante } \\
\text { Até } 4 \text { cigarros/dia } \\
\text { Mais } 4 \text { cigarros / dia }\end{array}$ & $\begin{array}{r}187 \\
22 \\
03 \\
07\end{array}$ & $\begin{array}{r}85,4 \\
10,0 \\
1,4 \\
3,2\end{array}$ & $\begin{array}{r}77 \\
06 \\
- \\
-\end{array}$ & $\begin{array}{r}92,8 \\
7,2 \\
- \\
-\end{array}$ \\
\hline Etilismo & $\begin{array}{l}\text { Não } \\
\text { Sim }\end{array}$ & $\begin{array}{r}166 \\
52\end{array}$ & $\begin{array}{l}76,1 \\
23,9\end{array}$ & $\begin{array}{l}66 \\
17\end{array}$ & $\begin{array}{l}79,5 \\
20,5\end{array}$ \\
\hline
\end{tabular}

* respostas válidas, excluídas as respostas ignoradas

Tabela 3 - Características do trabalho profissional dos trabalhadores de enfermagem de um hospital público em Feira de Santana, Bahia, 2010

\begin{tabular}{|c|c|c|c|c|c|}
\hline \multirow[t]{2}{*}{ Características do trabalho } & & \multicolumn{2}{|c|}{$\begin{array}{l}\text { Técnicos / Aux. } \\
\text { de enfermagem }\end{array}$} & \multicolumn{2}{|c|}{ Enfermeiros } \\
\hline & & $\mathbf{n}$ & $\%$ & $\mathbf{n}$ & $\%$ \\
\hline Tempo de trabalho na Enfermagem & $\begin{array}{l}0-5 \text { anos } \\
6-15 \text { anos } \\
16-25 \text { anos } \\
\text { Acima de } 26 \text { anos }\end{array}$ & $\begin{array}{l}82 \\
63 \\
37 \\
41\end{array}$ & $\begin{array}{l}36,8 \\
28,3 \\
16,6 \\
18,4\end{array}$ & $\begin{array}{l}40 \\
23 \\
12 \\
10\end{array}$ & $\begin{array}{l}47,1 \\
27,1 \\
14,1 \\
11,8\end{array}$ \\
\hline Turno de trabalho & $\begin{array}{l}\text { Diurno } \\
\text { Noturno } \\
\text { Outros }\end{array}$ & $\begin{array}{l}89 \\
51 \\
15\end{array}$ & $\begin{array}{r}57,4 \\
32,9 \\
9,7\end{array}$ & $\begin{array}{l}27 \\
08 \\
13\end{array}$ & $\begin{array}{l}56,3 \\
16,7 \\
27,1\end{array}$ \\
\hline Número de locais de trabalho & $\begin{array}{l}01 \\
02 \\
03 \\
04 \text { ou mais }\end{array}$ & $\begin{array}{r}123 \\
85 \\
11 \\
03\end{array}$ & $\begin{array}{r}55,4 \\
38,3 \\
5,0 \\
1,4\end{array}$ & $\begin{array}{l}19 \\
49 \\
14 \\
02\end{array}$ & $\begin{array}{r}23,6 \\
58,3 \\
16,7 \\
2,4\end{array}$ \\
\hline Tempo de deslocamento até o local de trabalho & $\begin{array}{l}\text { Até } 30 \mathrm{~min} \\
30 \mathrm{~min}-2 \mathrm{~h} \\
\text { Acima de } 2 \mathrm{~h}\end{array}$ & $\begin{array}{r}93 \\
110 \\
16\end{array}$ & $\begin{array}{r}42,5 \\
50,2 \\
7,3\end{array}$ & $\begin{array}{l}71 \\
09 \\
04\end{array}$ & $\begin{array}{r}84,5 \\
10,7 \\
4,8\end{array}$ \\
\hline Renda mensal & $\begin{array}{l}1 \text { a } 3 \text { salários }(\mathrm{SM}) \\
4-7 \text { salários }(\mathrm{SM})\end{array}$ & $\begin{array}{r}195 \\
02\end{array}$ & $\begin{array}{r}99,0 \\
1,0\end{array}$ & $\begin{array}{l}58 \\
22\end{array}$ & $\begin{array}{l}72,5 \\
27,5\end{array}$ \\
\hline
\end{tabular}

* respostas válidas, excluídas as respostas ignoradas

SM = Salário mínimo

As características profissionais são apresentadas na Tabela 3. Com relação ao tempo de trabalho na Enfermagem, os profissionais apresentaram, em média, 11,8 anos $\pm 9,9$ de trabalho, sendo que a maioria informou até 05 anos, dentre os quais $36,8 \%$ (82) são técnicos e auxiliares e $47,1 \%$ (40) enfermeiros.

O turno de trabalho mais frequente foi o diurno, não apresentando diferenças entre as categorias. No entanto, a frequência de trabalho noturno foi superior entre os técnicos e auxiliares. Destaca-se que, neste hospital, no período noturno, ocorre redução do quantitativo de enfermeiros e a maioria assume dois ou mais setores neste período, fator que pode aumentar sobrecarga física e mental (Tabela 3).

Estudos apontam que o trabalho noturno, o alternado e os plantões são muito nocivos à saúde dos profissionais, levando a alterações nos padrões do sono, nas funções gastrintestinais e no ritmo circadiano, dificultando a conciliação entre a vida 
familiar e social ${ }^{(6)}$. Dessa forma, considera-se que o déficit de sono reduz a capacidade cognitiva, diminui a capacidade de execução de tarefas e expõe os trabalhadores de enfermagem a acidentes e falhas. Observa-se que o regime de trabalho, a depender do tipo, pode ser considerado como um fator de risco para o desenvolvimento de agravos a saúde.

Duplo emprego foi uma realidade para 58,3\% (48) dos enfermeiros; já a maioria dos técnicos e auxiliares da instituição em estudo não apresentava outro vínculo empregatício, 55,4\% (123). Esses resultados corroboram com a realidade vivenciada por $50,3 \%$ das trabalhadoras de enfermagem de um hospital universitário de São Paulo ${ }^{(7)}$. Na atual realidade econômica dos trabalhadores da saúde é comum a dupla e até tripla jornada de trabalho, em virtude dos baixos salários, os quais são insuficientes para o sustento adequado da família (Tabela 3).

O efeito da dupla jornada na saúde destes profissionais é marcante, em especial entre as mulheres, que tendem a agregar a realização do trabalho doméstico e o cuidado com os filhos. Essa duplicidade de papéis repercute no seu cotidiano, podendo levar a diminuição do rendimento no trabalho e do tempo dispensado ao autocuidado e lazer. Além disso, por conta dessa sobrecarga estes profissionais tem maior tendência ao adoecimento e absenteísmo ${ }^{(8)}$.

\section{Perfil de adoecimento dos trabalhadores de enfermagem}

A presença de doenças ocupacionais diagnosticadas por médico do trabalho foi informada por $14,3 \%$ (44) da população estudada. Entre os enfermeiros, 7,1\% (06) referiram alguma doença, frequência que aumenta para 17,2\% (38) entre os técnicos e auxiliares.

Na Enfermagem, a ocorrência de maior quantidade de relatos de adoecimento entre o pessoal de nível médio talvez se explique pela natureza do trabalho desenvolvido: assistência direta ao cliente, como banho, troca de roupas, transferências e administração de medicamentos, tarefas que exigem maior esforço físico, repetitivas e monótonas, além do contato muito próximo com o sofrimento ${ }^{(9)}$. Estudo realizado em Minas Gerais evidenciou que auxiliares e técnicos de enfermagem foram os que mais demandaram atendimento médico $86,9 \%$, seguidos pelos enfermeiros, com $10,9 \%$ dos atendimentos ${ }^{(10)}$.

Entre os problemas de saúde referidos desde o início de suas atividades laborais na unidade hospitalar, 52,8\% (163) apontaram a lombalgia como mais frequente, seguida por varizes em membros inferiores 46,3\% (143) e infecção urinária 37,5\% (116). Estes problemas de saúde não variaram quando separados por categorias profissionais. No entanto, entre os enfermeiros, as maiores frequências de agravos foram varizes em membros inferiores 47,1\% (40), e infecções respiratórias repetidas $20 \%$ (17). Entre os técnicos / auxiliares de enfermagem os demais problemas apresentaram prevalências superiores (Tabela 4).

Os dados desta pesquisa são inferiores aos encontrados por outros autores, em estudo realizado em uma UTI de Ribeirão Preto-SP, onde a lombalgia acometeu $67 \%$ dos profissionais de enfermagem entrevistados ${ }^{(11)}$.

A presença de varizes de membros inferiores encontrou elevada prevalência, provavelmente em razão dos longos períodos na posição ortostática e das longas distâncias percorridas no decorrer do trabalho; no entanto, o percentual foi inferior ao estudo realizado em Hospital Público de Curitiba-PR, em 2008 , onde a prevalência dessa afecção foi de $57,23 \%{ }^{(12)}$.

Tabela 4 - Problemas de saúde referidos pelos trabalhadores de enfermagem de um hospital público de Feira de Santana, Bahia, 2010

\begin{tabular}{|c|c|c|c|}
\hline Problemas de Saúde & Sim & Não & Prev. \\
\hline Lombalgias & & & 52,6 \\
\hline $\begin{array}{l}\text { Técnicos/ Aux. de enfermagem } \\
\text { Enfermeiros }\end{array}$ & $\begin{array}{l}120 \\
42\end{array}$ & $\begin{array}{c}103 \\
43\end{array}$ & $\begin{array}{l}53,8 \\
49,4\end{array}$ \\
\hline Varizes MMII & & & 46,1 \\
\hline $\begin{array}{l}\text { Técnicos/ Aux. de enfermagem } \\
\text { Enfermeiros }\end{array}$ & $\begin{array}{c}102 \\
40\end{array}$ & $\begin{array}{c}121 \\
45\end{array}$ & $\begin{array}{l}45,7 \\
47,1\end{array}$ \\
\hline Infecção urinária & & & 37,7 \\
\hline $\begin{array}{l}\text { Técnicos/ Aux. de enfermagem } \\
\text { Enfermeiros }\end{array}$ & $\begin{array}{l}89 \\
27\end{array}$ & $\begin{array}{c}134 \\
58\end{array}$ & $\begin{array}{l}39,9 \\
31,8\end{array}$ \\
\hline Hipertensão & & & 19,8 \\
\hline $\begin{array}{l}\text { Técnicos/ Aux. de enfermagem } \\
\text { Enfermeiros }\end{array}$ & $\begin{array}{l}51 \\
10\end{array}$ & $\begin{array}{c}172 \\
75\end{array}$ & $\begin{array}{l}22,9 \\
11,8\end{array}$ \\
\hline Lesão por esforço repetitivo & & & 15,9 \\
\hline $\begin{array}{l}\text { Técnicos/ Aux. de enfermagem } \\
\text { Enfermeiros }\end{array}$ & $\begin{array}{c}40 \\
9\end{array}$ & $\begin{array}{c}183 \\
76\end{array}$ & $\begin{array}{l}17,9 \\
10,6\end{array}$ \\
\hline Infecções respiratórias repetidas & & & 16,2 \\
\hline $\begin{array}{l}\text { Técnicos/ Aux. de enfermagem } \\
\text { Enfermeiros }\end{array}$ & $\begin{array}{l}33 \\
17\end{array}$ & $\begin{array}{c}190 \\
68\end{array}$ & $\begin{array}{l}14,8 \\
20,0\end{array}$ \\
\hline Sinusite crônica & & & 14,3 \\
\hline $\begin{array}{l}\text { Técnicos/ Aux. de enfermagem } \\
\text { Enfermeiros }\end{array}$ & $\begin{array}{l}32 \\
12\end{array}$ & $\begin{array}{c}191 \\
73\end{array}$ & $\begin{array}{l}14,3 \\
14,1\end{array}$ \\
\hline
\end{tabular}

* respostas válidas, excluídas as respostas ignoradas

Outra afecção bastante relatada foi a infecção urinária que, para os trabalhadores de enfermagem, pode estar relacionada a alterações no ritmo circadiano (trabalho em turnos), ambientes com temperaturas elevadas, exposição a gases e a substâncias químicas, assim como ao excesso de atividades. Esses fatores podem ser agravados por descuido na atenção às necessidades básicas de alimentação/eliminação e pela anatomia feminina.

A presença de Hipertensão Arterial também foi relatada pelos profissionais, sendo cerca de 2 (duas) vezes maior entre os técnicos e auxiliares quando comparado aos enfermeiros. Tais dados, no entanto foram inferiores aos encontrados em estudo realizado em Salvador-BA, que encontrou 40,1\% de HAS em auxiliares de enfermagem ${ }^{(7)}$; e em Fortaleza-CE, que identificou uma prevalência de $43,1 \%$ de HAS na mesma categoria profissional ${ }^{(13)}$.

As queixas osteomusculares foram as mais referidas neste estudo. Os enfermeiros apresentaram a prevalência mais elevada, exceto para a dor nos braços (Tabela 5). Este resultado é contrário ao observado em outro estudo ${ }^{(10)}$. A maior frequência destes sintomas entre os técnicos e auxiliares de enfermagem pode estar associada à execução da assistência direta 
ao cliente, a realização de movimentos repetitivos, aliados a exigências de força.

Os sintomas relacionados ao aparelho respiratório 33,0\% (94) apontaram rinite; e dor de garganta, com 28,2\% (80) dos relatos. Quando separados por categoria profissional os enfermeiros apresentaram maiores prevalência para os sintomas de rinite $33,3 \%(27)$, tosse $14,4 \%$ (12) e falta de ar 8,5\% (07) (Tabela 5). As infecções respiratórias têm sido pouco valorizadas pelos trabalhadores de saúde, provavelmente por que demandam poucas repercussões físicas e poucas faltas nas suas atividades laborais.

Estes profissionais acreditam que as afecções respiratórias podem ser provocadas por alterações climáticas ou baixa resistência, raramente relacionando-as à sobrecarga de trabalho, à má alimentação. às condições inadequadas de trabalho e aos fatores de risco laborais. Ressalte-se que os agentes químicos podem causar também o comprometimento respiratório ${ }^{(6)}$.

Com relação aos agravos relacionados ao sistema digestório, as prevalências foram semelhantes nos dois grupos profissionais; Acredita-se que problemas digestivos, como a gastrite nervosa, pode ser consequência do ambiente laboral estressante, em especial em ambiente hospitalar, onde é comum a falta de recursos materiais e humanos, que dificultam a prestação adequada dos cuidados de enfermagem ${ }^{(6)}$. Para esta queixa os enfermeiros apresentaram prevalências superiores às apresentadas pelos técnicos e auxiliares de enfermagem.

As perturbações gastrointestinais, como úlceras pépticas e duodenais, gastroduodenites, anorexia e obstipação intestinal também podem ser ocasionadas por alterações no ritmo circadiano em decorrência do trabalho em turnos ${ }^{(6,14-15)}$.

Os resultados relacionados à saúde mental apontaram prevalência de 47,0\% (131) para o cansaço mental, 33,7\% (93) nervosismo e $24,3 \%$ (68) esquecimento frequente, sendo os enfermeiros mais acometidos por todos estes sintomas (Tabela 5). A ocorrência destes sintomas são condizentes com dados encontrados em outra pesquisa, onde o cansaço mental apresentou frequência de $40 \%$, o esquecimento $27,9 \%$. Estes eventos podem ocorrer como consequência da sobrecarga de trabalho, trabalho em turnos e altos níveis de exigência na execução das tarefas ${ }^{(16)}$.

A prevalência de Distúrbios Psíquicos Menores (DPM) foi de $26,8 \%$ entre técnicos e auxiliares de enfermagem e de $32,1 \%$ entre os enfermeiros. $\mathrm{O}$ estudo apontou alta prevalência de sofrimento mental, em especial nos enfermeiros, sendo este índice superior ao obtido em outros estudos com trabalhadores de enfermagem e outros trabalhadores de saúde ${ }^{(5,7,14,16)}$.

Nesse estudo foram encontrados elevadas frequências de trabalho de alta exigência, em especial entre os técnicos e auxiliares de enfermagem $16,6 \%$ (32) quando comparado com os enfermeiros 3,8\% (03). A influência dos fatores psicossociais e a organização do trabalho em saúde tem revelado associação entre piores condições de trabalho com sintomas físicos e emocionais ${ }^{(17)}$.

Dentre os problemas relacionados com o sono $36,6 \%$ (101) referiram sonolência e 21,8\% (61) insônia. Entre os enfermeiros a prevalência destes sintomas foi maior quando comparada com os técnicos e auxiliares (Tabela 5).
Tabela 5 - Queixas e sintomas de alguns agravos à saúde referidos pelos trabalhadores de enfermagem de um hospital público em Feira de Santana, Bahia, 2010

\begin{tabular}{lccc}
\hline Queixas e Sintomas & Sim & Não & Prev. \\
\hline Sintomas Osteomusculares & & & \\
& & & \\
Dor nas pernas & & & 66,4 \\
$\quad$ Técnicos/ Aux. de enfermagem & 134 & 72 & 65,0 \\
$\quad$ Enfermeiros & 58 & 25 & 69,9 \\
& & & 62,0 \\
Dor nas costas & 126 & 82 & 60,6 \\
$\quad$ Técnicos/ Aux. de enfermagem & 52 & 27 & 65,8 \\
$\quad$ Enfermeiros & & & 38,2 \\
& & & \\
Dor nos braços & 86 & 118 & 42,2 \\
$\quad$ Técnicos/ Aux. de enfermagem & 24 & 60 & 28,6 \\
$\quad$ Enfermeiros & & & 35,4 \\
$\quad$ Formigamento nas pernas & & & \\
$\quad$ Técnicos/ Aux. de enfermagem & 70 & 130 & 35,0 \\
$\quad$ Enfermeiros & 29 & 51 & 36,3
\end{tabular}

\section{Sintomas Respiratórios}

\begin{tabular}{|c|c|c|c|}
\hline Alergias & & & 35,3 \\
\hline Técnicos/ Aux. de enfermagem & 73 & 121 & 37,6 \\
\hline Enfermeiros & 24 & 57 & 29,6 \\
\hline Rinite & & & 32,7 \\
\hline Técnicos/ Aux. de enfermagem & 66 & 137 & 32,5 \\
\hline Enfermeiros & 27 & 54 & 33,3 \\
\hline Dor na garganta & & & 27,9 \\
\hline Técnicos/ Aux. de enfermagem & 60 & 141 & 29,9 \\
\hline Enfermeiros & 19 & 63 & 23,2 \\
\hline Tosse & & & 13,1 \\
\hline Técnicos/ Aux. de enfermagem & 24 & 167 & 12,6 \\
\hline Enfermeiros & 12 & 71 & 14,5 \\
\hline Rouquidão & & & 9,0 \\
\hline Técnicos/ Aux. de enfermagem & 18 & 178 & 9,2 \\
\hline Enfermeiros & 07 & 75 & 8,5 \\
\hline Perda temporária da voz & & & 4,0 \\
\hline Técnicos/ Aux. de enfermagem & 10 & 181 & 5,2 \\
\hline Enfermeiros & 01 & 80 & 1,2 \\
\hline Falta de ar & & & 5,8 \\
\hline Técnicos/ Aux. de enfermagem & 09 & 185 & 4,6 \\
\hline Enfermeiros & 07 & 75 & 8,5 \\
\hline
\end{tabular}

\section{Sintomas Digestórios}

Problemas digestivos

Técnicos/ Aux. de enfermagem Enfermeiros

$\begin{array}{ccc} & & 21,0 \\ 41 & 164 & 20,0 \\ 19 & 62 & 23,5\end{array}$

\section{Sintomas Relacionados à Saúde Mental}

$\begin{array}{lccc}\text { Cansaço mental } & & & 47,1 \\ \text { Técnicos/ Aux. de enfermagem } & 82 & 113 & 42,1 \\ \text { Enfermeiros } & 49 & 34 & 59,0\end{array}$

Continua 
Tabela 5 (cont.)

\begin{tabular}{lccc}
\hline Queixas e Sintomas & Sim & Não & Prev. \\
\hline $\begin{array}{l}\text { Nervosismo } \\
\quad \text { Técnicos/ Aux. de enfermagem }\end{array}$ & 62 & 131 & 32,1 \\
$\quad$ Enfermeiros & 31 & 51 & 37,8 \\
Esquecimento & & & 24,4 \\
$\quad$ Técnicos/ Aux. de enfermagem & & & 24,1 \\
$\quad$ Enfermeiros & 47 & 148 & 24,0
\end{tabular}

\section{Sintomas Relacionados ao Sono}

$\begin{array}{lccc}\text { Sonolência } & & & 36,4 \\ \quad \text { Técnicos/ Aux. de enfermagem } & 67 & 125 & 34,9 \\ \quad \text { Enfermeiros } & 33 & 50 & 39,8 \\ & & & 21,5 \\ \text { Insônia } & & & 21,4 \\ \quad \text { Técnicos/ Aux. de enfermagem } & 42 & 154 & 21,4 \\ \quad \text { Enfermeiros } & 18 & 65 & 21,7\end{array}$

Sint. Relacionados aos órgãos dos sentidos

$\begin{array}{lccc}\text { Irritação nos olhos } & & & 14,6 \\ \quad \text { Técnicos/ Aux. de enfermagem } & 32 & 168 & 16,0 \\ \text { Enfermeiros } & 09 & 71 & 11,3 \\ & & & 9,6 \\ \text { Zumbido no ouvido } & & & 9,4 \\ \quad \text { Técnicos/ Aux. de enfermagem } & 18 & 174 & 9,4 \\ \quad \text { Enfermeiros } & 08 & 72 & 10,0 \\ & & & 6,3 \\ \text { Não ouve bem } & 13 & 175 & 6,9 \\ \quad \text { Técnicos/ Aux. de enfermagem } & 04 & 76 & 5,0 \\ \quad \text { Enfermeiros } & & & \end{array}$

\section{Sintomas Inespecíficos}

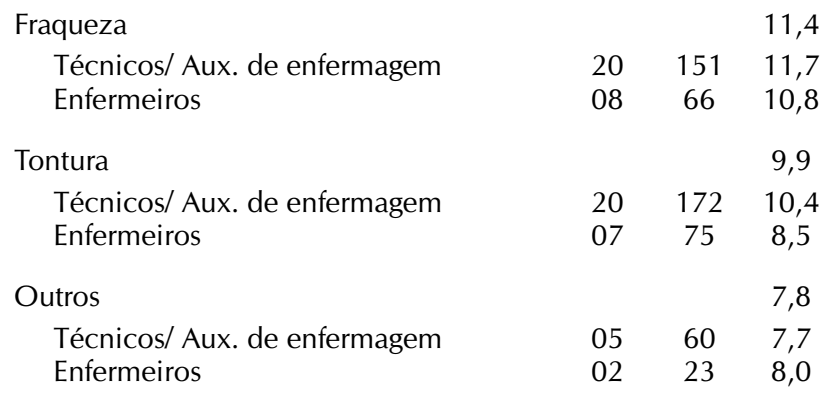

Na análise do Mine-Sleep Questionnare verificamos que ambas as categorias apresentam padrões elevados de sono muito alterado, enfermeiros com 43,4\% (36) e técnicos/auxiliares $44,9 \%$ (96). No entanto, os trabalhadores de nível médio apresentaram maiores prevalências de sono moderadamente alterado $13,7 \%$ (29), enquanto os enfermeiros relataram maiores escores de sono bom.

Corroborando estes achados, alguns estudos apontam que uma noite de privação de sono acarreta sintomas como insônia e sonolência excessiva durante o dia ${ }^{(18)}$; além disso, o trabalhador de turno tem maior risco de desenvolver problemas cardiovasculares, gastrointestinais e outras afecções.
A irritabilidade e a falta de memória e de concentração são apontadas como outros problemas decorrentes da privação do sono ${ }^{(18)}$.

As alterações relativas aos órgãos dos sentidos foram relatadas por alguns autores e podem ser consequência da exposição a substâncias nocivas no ambiente laboral, como gases anestésicos, produtos químicos para limpeza e desinfecção de materiais e de superfícies, e ação de agentes biológicos (contaminação pelas mãos) ${ }^{(16)}$.

Observa-se que os agravos à saúde do sistema respiratório e dos órgãos dos sentidos podem estar relacionados a infecções hospitalares, por meio de contaminação, ambientes fechados e pouco ventilados, baixa imunidade e estresse, fatores de risco presentes no cotidiano destes trabalhadores ${ }^{(15)}$.

Os sintomas inespecíficos estudados foram classificados como tonturas, fraqueza e outros, que geralmente indicam que algo não está funcionando bem, podendo estar associados a outros agravos a saúde. Cerca de 19\% dos agravos a saúde apresentados por trabalhadores hospitalares relacionavam-se a enfermidades psicossomáticas, "poliqueixas" e sintomas mal definidos ${ }^{(14)}$.

\section{CONCLUSÕES}

O presente estudo possibilitou a identificação de inúmeros agravos à saúde referidos pela equipe de enfermagem, muitos dos quais podem ser decorrentes das cargas presentes no ambiente laboral. A Enfermagem é reconhecida por ser uma atividade penosa, que lida diretamente com o sofrimento, o que exige muito, física e emocionalmente, do trabalhador. Soma-se a isso o frequente déficit de profissionais nas unidades, os turnos prolongados, as condições inadequadas de trabalho, limitado poder de decisão, entre outros fatores que contribuem para o processo de desgaste destes trabalhadores.

Evidenciamos neste estudo que os enfermeiros queixaram-se mais de sintomas osteomusculares, sintomas relacionados à saúde mental, sintomas digestórios e problemas com o sono, isso pode estar associado não apenas a sobrecarga física, mas principalmente a sobrecarga psíquica, visto que, estes profissionais são responsáveis pelo bom andamento das unidades (material, equipamentos, realização de exames) e também pela supervisão de atividades realizadas pelo pessoal de nível médio e algumas vezes pelas atividades desenvolvidas pelo pessoal de nível superior.

Entre técnicos e auxiliares de enfermagem as queixas mais frequências estavam relacionadas a agravos do sistema respiratório, o que pode ser justificado pelo contato mais frequente e próximo ao paciente.

Por fim, recomenda-se que os trabalhadores de enfermagem incorporem ao seu cotidiano atividades preventivas e ampliem a consciência do seu processo de trabalho e do desgaste decorrente deste, adquirindo conhecimento científico para prevenção dos agravos à saúde e fomentando o fortalecimento da categoria para lutar por melhores condições de trabalho e de vida. 


\section{REFERÊNCIAS}

1. Ministério da Saúde (BR), Organização Pan Americana da Saúde no Brasil. Doenças relacionadas ao trabalho: manual de procedimentos para os serviços de saúde [Internet]. Brasília (DF): Ministério da Saúde; 2001 [acesso em 30 de janeiro de 2013]. Disponível em: http://www. acervo.epsjv.fiocruz.br/beb/textocompleto/001418

2. Kirchhof ALC, Magnago TSBS, Camponogara S, Griep $\mathrm{RH}$, Tavares JP, Prestes FC, et al. Condições de trabalho e características sócio demográficas relacionadas à presença de distúrbios psíquicos menores em trabalhadores de enfermagem. Texto \& Contexto Enferm [Internet]. 2009 [acesso em 30 de janeiro de 2013];18(2):215-23. Disponível em: http://www.scielo.br/scielo.php?script=sci_artt ext\&pid $=$ S0104-07072009000200003

3. Elias MA, Navarro VL. A relação entre o trabalho, a saúde e as condições de vida: negatividade e positividade no trabalho das profissionais de enfermagem de um hospital escola. Rev Latinoam Enferm [Internet]. 2006 [acesso em 30 de janeiro de 2013];14(4):517-25. Disponível em: http://www.scielo.br/scielo.php?pid=S0104$-11692006000400008 \&$ script $=$ sci_abstract $\&$ tlng $=$ pt

4. Theme Filha MM, Costa MASC, Guilam MCR. Estresse ocupacional e autoavaliação de saúde entre profissionais de enfermagem. Rev Latinoam Enferm [Internet]. 2013 [acesso em 30 de janeiro de 2013];21(2). Disponível em: http://www.scielo.br/scielo.php?pid=S0104$-11692013000200475 \&$ script $=$ sci_arttext\&tlng $=$ pt

5. Meneghini F, Paz AA, Lautert L. Fatores ocupacionais associados aos componentes da Síndrome de Bournout em trabalhadores de enfermagem. Texto \& Contexto Enferm [Internet]. 2011 [acesso em 30 de janeiro de 2013];20(2):22533. Disponível em: http://www.scielo.br/scielo.php?pid= S0104-07072011000200002\&script =sci arttext

6. Bulhões I. Riscos do Trabalho de Enfermagem. 2. ed. Rio de Janeiro (RJ): Ática; 1998.

7. Silva AA, Rotenberg L, Fischer FM. Jornadas de trabaIho na enfermagem: entre necessidades individuais e condições de trabalho. Rev Saúde Pública [Internet]. 2011 [acesso em 19 de junho de 2011];45(6). Disponível em: http://www.scielo.br/scielo.php?pid=S0034$-89102011000600014 \&$ script $=$ sci_arttext

8. Rezende MP. Agravos a saúde de auxiliares de enfermagem resultantes da exposição ocupacional aos riscos físicos [dissertação]. Ribeirão Preto (SP): Universidade de São Paulo; 2003.

9. Nascimento GM. Estudo do absenteísmo dos trabalhadores de enfermagem em uma unidade básica e distrital de saúde do município de Ribeirão Preto-SP [dissertação]. Ribeirão Preto (SP): Universidade de São Paulo; 2003.

10. Murofuse NT, Marziale MHP. Doenças do sistema osteomuscular em trabalhadores de enfermagem. Rev Latinoam Enferm [Internet]. 2005 [acesso em 30 de janeiro de 2013];13(3):364-73. Disponível em: http:// www.scielo.br/scielo.php?script $=$ sci_arttext\&pid =S0104-11692005000300011

11. Petersen RS, Marziale MHP. Lombalgia caracterizada pela resistência da musculatura e fatores ocupacionais associados à enfermagem. Rev Latinoam Enferm [Internet]. 2014 [acesso em 30 de janeiro de 2013];22(3). Disponível em: http://www.scielo.br/pdf/rlae/v22n3/pt_0104-1169rlae-22-03-00386.pdf

12. Maynardes DC, Sarquis LMM, Kirchhof ALC. Trabalho noturno e morbidade de trabalhadores de enfermagem. Cogitare Enferm [Internet]. 2009 [acesso em 30 de janeiro de 2013];14(4):703-8. Disponível em: http://bases.bireme.br/cgi-bin/wxislind.exe/iah/online/?lsisScript $=$ iah/iah. $\mathrm{xis} \& \mathrm{src}=$ google $\&$ base $=$ LILACS\&lang $=p \&$ nextAction $=\mathrm{I}$ $\mathrm{nk} \&$ exprSearch $=568370$ \&indexSearch $=$ ID

13. Custódio IL, Lima FLT, Almeida MI, Silva LF, Monteiro ARM. Perfil sociodemográfico e clínico de uma equipe de enfermagem portadora de Hipertensão Arterial. Rev Bras Enferm. 2011;64(1):18-24.

14. Pitta AM. Hospital: dor e morte como ofício. São Paulo (SP): Hucitec; 1990.

15. Barboza DB, Soler ZA. Afastamento do trabalho na enfermagem: ocorrências com trabalhadores de um hospital de ensino. Rev Latinoam Enferm. 2007;11(2):177-83.

16. Souza MNM, Martins Júnior DF, Silva MV, Costa JÁ, Nascimento Sobrinho CL. Trabalho e saúde dos profissionais de enfermagem de um hospital especializado de Feira de Santana, Bahia. Rev Baiana Saúde Pública [Internet]. 2011 [acesso em 30 de janeiro de 2013];35(1):38-54. Disponível em: http://inseer.ibict.br/rbsp/index.php/rbsp/ article/viewFile/146/141

17. Gholamzadeh S, Sharif F, Rad FD. Sources of occupational stress and coping strategies among nurses who are working in Admission and Emergency Department in Hospitals affiliated to Shiraz University of Medical Sciences, Iran. Iran J Nurs Midwifery Res [Internet]. 2011 [cited 2013 January 30];16(1):42-7. Available from: http:// www.ncbi.nlm.nih.gov/pubmed/21772921

18. Garay $\mathrm{CH}$. Distúrbios do sono devidos ao trabalho em turnos. In: Reimão R. Sono: estudo abrangente. 2. ed. São Paulo (SP): Atheneu; 1996. p. 390-91. 